\title{
Extracorporeal Membrane Oxygenation in Cardiac Intensive Care Unit
}

\author{
Venkat Goyal ${ }^{1}$ Pranay Oza ${ }^{1}$ \\ ${ }^{1}$ Riddhi Vinayak Critical Care and Cardiac Centre, Mumbai, \\ Maharashtra, India \\ J Card Crit Care TSS 2017;1:10-14.
}

Address for correspondence Dr. Venkat Goyal, Department of Cardiology, Riddhi Vinayak Critical Care and Cardiac Centre, 559/1, Riddhi Vinayak Temple lane, S V Road, Malad West, Mumbai, Maharashtra 400064, India (e-mail: ecmosocietyofindia@hotmail.com).

\begin{abstract}
Keywords

- extracorporeal membrane oxygenation

- cardiopulmonary arrest

- catecholamine

- left ventricular assist device

In critical care units, doctors usually witness patients coming with single organ failure and subsequently suffer multiorgan failure before they succumb to the destiny. It is well-known that hardly a few patients die of single organ failure, and with addition of every organ, the risk of mortality increases by $10 \%$. The multiorgan failure is secondary to inadequate organ function, tissue perfusion, and oxygenation or due to iatrogenic causes. Extracorporeal membrane oxygenation (ECMO) is not a treatment by itself but a mechanical assist device or rather a replacement therapy to sustain life, to give rest to the organs, and to maintain adequate perfusion and oxygenation. There are various articles discussing the outcomes of ECMO in cardiogenic shock with varied etiology. ECMO support can rescue $40 \%$ of patients with otherwise fatal cardiogenic shock (mortality without ECMO is $>80 \%$ ). As per ELSO data January 2017, 10,982 patients were reported in adult cardiac ECMO, out of whom $56 \%$ survived ECLS and $\quad 40 \%$ survived to discharge. The newer scoring system named SAVE score (its online calculator [www.save-score.com]) offers a validated tool to predict survival for patients receiving ECMO for refractory cardiogenic shock.
\end{abstract}

\section{Introduction}

Extracorporeal membrane oxygenation (ECMO) is a modified form of heart-lung bypass, used to treat acutely ill patients with life-threatening but potentially reversible diseases. It is temporary, but it provides prolonged support of circulatory, respiratory, and other organ systems done outside the operating room (OR) in intensive care unit (ICU) setting. It helps sustain life in case of cardiopulmonary arrest, provides gas exchange, and maintains mean arterial pressure and tissue perfusion. Thus, it prevents hypoxic and hypercapnic insult to the organs, decreases inotropic requirement thereby reducing their toxic effect, decreases ventilatory requirement thereby reducing ventilator-induced lung injury (VILI), and gives rest to the lungs and heart.

In critical care units, doctors usually witness patients coming with single organ failure and subsequently suffer multiorgan failure before they succumb to the destiny. It is well-known that hardly a few patients die of single organ failure, and with addition of every organ, the risk of mortality increases by $10 \%$. The multiorgan failure is secondary to inadequate organ function, tissue perfusion, and oxygenation or due to iatrogenic causes. Iatrogenic causes include VILI, fluid overload and organ edema needed to support cardiovascular function, worsening global or regional tissue perfusion from the adverse effects of catecholamines, and finally secondary sepsis. All existing modalities in critical care unit such as ventilator, intra-aortic balloon pump (IABP), inotropes, etc. are support systems and the replacement therapies. It is like slogging a dead horse and no actual rest to organs. ECMO is the only modality that replaces the heart and lung function, thereby giving rest to them.

ECMO in its initial phase got well established in neonatal and pediatric group, and later from 2009 onward, it also got some popularity in adult respiratory ECMO and now the number of its users is increasing. Another place where ECMO
DOI https://doi.org/ $10.1055 / \mathrm{s}-0037-1605345$
Copyright (๑) 2017 Official Publication of License terms The Simulation Society (TSS), accredited by International Society of Cardiovascular Ultrasound (ISCU) 
is still underutilized is in adult cardiac ICU. The incidences of cardiogenic shock (CS) due to varied etiology are increasing in both developed and developing countries.

According to SHOCK registry, the overall incidence of shock complicating acute myocardial infarction (MI) is 5 to $7 \%$. The main causes of CS in acute MI include left ventricular (LV) failure (79\%), severe mitral regurgitation (MR) (7\%), and ventricular septal defect (VSD) (4\%). The in-hospital mortality of CS is around $60 \%$. The median time from MI to onset of CS is 5.5 hours and $75 \%$ of patients develop shock within 24 hours. ${ }^{1}$ As per SHOCK trial, there was no significant difference in 30-day mortality ( 46.7 vs. $56 \%, p=0.11$ ) between the CS patient who were managed with immediate revascularization and those managed on medical therapy only. However, there was significant decrease in all-cause mortality at 6 months ( 50.3 vs. $63.1 \% p=0.027$ ). Another SHOCK II trial on CS and IABP fails to show 30-day mortality benefits of using IABP in CS. At 30 days, 119 patients in the IABP group (39.7\%) and 123 in the control group $(41.3 \%)$ died $(p=0.69){ }^{2}$ As for the patient of CS secondary to MI or any other causes, doctors do not have much to offer. Mechanical circulatory support is emerging as better alternative with survival rate around $40 \%$. Among all mechanical circulatory supports, ECMO remains the most economical and easiest to initiate.

\section{Indications, Contraindications, and Criteria for ECMO in Cardiac ICU}

ECMO is not a treatment by itself but a mechanical assist device or rather a replacement therapy to sustain life, give rest to the organs, and to maintain adequate perfusion and oxygenation. In cardiac ICU, it is indicated in any kind of CS. Shock that persists despite adequate fluid resuscitation, inotropes, and vasoconstrictor with or without IABP becomes the indication of ECMO. ECMO has been successfully used as a bridge to myocardial recovery, cardiac transplantation, or implantation of a left ventricular assist device (LVAD) in patients with various etiologies of overt cardiac failure, such as acute $\mathrm{MI},{ }^{3}$ end-stage dilated cardiomyopathy, ${ }^{4}$ viral or toxic myocarditis, ${ }^{5}$ complications of cardiac surgery, ${ }^{6}$ or

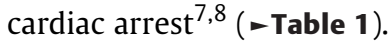

The selection criterion to initiate ECMO in CS is progressive deterioration in hemodynamic profile with indications of impending multiorgan system failure due to poor tissue perfusion (e.g., metabolic acidosis, oliguria, lactate rising). It is important to intervene early, before the onset irreversible damage secondary to support therapy and shock per se. ${ }^{9}$ Rather than one particular number, the progress in 6 to 8 hours is an important factor for determining whether the patient needs ECMO or not (-Tables $2, \mathbf{3}$ ).

The patient with unrecoverable heart and not a candidate for transplant or VAD and chronic organ dysfunction (chronic obstructive pulmonary disease [COPD], cirrhosis, renal failure, end-stage malignancy) remains as contraindication for ECMO, though the other relative contraindications include advanced age ( $>75$ years), anticoagulation and prolonged shock as indicated by low blood pressure, metabolic acidosis base excess $>5$ for 12 hours, and oliguria $<0.5 \mathrm{~mL} / \mathrm{h}$ for $>12$ hours.

\section{Management}

The usual management of CS depends on the pathophysiology of shock and underlying cause per se. Depending on pathophysiology, if it is a preload failure, treat with the fluids, and if it is a pump failure, inotropes or inodilators are recommended. If it is a distributive shock, vasoconstrictors and fluids are recommended. Also, one has to optimize the heart rate and treat arrhythmias, if any. After initial therapy, the patient should be reassessed for end-organ function and cardiac output. Once the patient stabilizes, explore the possibility and

Table 1 Indications of ECMO in cardiac ICU

\begin{tabular}{|l|}
\hline - Acute refractory CS secondary to \\
\hline o Acute MI \\
\hline $\begin{array}{l}\text { o Poisoning-cardiotoxic drug overdose, calcium channel blockers, } \beta \text {-blockers, aluminum phosphide toxicity, tricyclic } \\
\text { antidepressant }\end{array}$ \\
\hline o Traumatic cardiac tamponade with persistent cardiac arrest, or myocardial contusion following ballistic chest trauma \\
\hline o Fulminant myocarditis \\
\hline o Postcardiotomy CS \\
\hline - Decompensated chronic heart failure \\
\hline - Cardiomyopathy, e.g., peripartum cardiomyopathy \\
\hline - Refractory malignant arrhythmias \\
\hline - Cardiac transplant - \\
\hline o Post transplant graft failure, with elevated pulmonary pressures and RA/LV dysfunction \\
\hline o Acute cardiac allograft rejection \\
\hline o Pretransplant stabilization \\
\hline - ECPR
\end{tabular}

Abbreviations: CS, cardiogenic shock; ECMO, extracorporeal membrane oxygenation; ECPR, extracorporeal cardiopulmonary resuscitation; ICU, intensive care unit; LV, left ventricular; MI, myocardial infarction; RA, right ventricular. 
Table 2 Selection criteria for ECMO in cardiac ICU

\begin{tabular}{|l|}
\hline Selection criteria \\
\hline $\begin{array}{l}\text { - Rising lactate of }>0.75 \mathrm{mmol} / \mathrm{L} \text { or lactate level } \\
>4 \mathrm{mmol} / \mathrm{L}\end{array}$ \\
\hline - Inotropic score $>40$ \\
\hline - Mean arterial pressure $<60$ with inotropic supports \\
\hline - Urine output $<0.5 \mathrm{~mL} / \mathrm{kg} / \mathrm{h}$ for $6-8 \mathrm{~h}$ \\
\hline - Cardiac index $<2 \mathrm{~L} / \mathrm{m}^{2} / \mathrm{min}$ for $3 \mathrm{~h}$ \\
\hline - Base deficit $>5$ for $3 \mathrm{~h}$ \\
\hline
\end{tabular}

Abbreviations: ECMO, extracorporeal membrane oxygenation; ICU, intensive care unit.

Table 3 Inotropic score

\begin{tabular}{|l|}
\hline $1 \mu \mathrm{g} / \mathrm{kg} / \mathrm{min}$ dopamine $=1$ point \\
\hline $1 \mu \mathrm{g} / \mathrm{kg} / \mathrm{min}$ dobutamine $=1$ point \\
\hline $0.01 \mu \mathrm{g} / \mathrm{kg} / \mathrm{min}$ adrenaline $=1$ point \\
\hline $0.5 \mu \mathrm{g} / \mathrm{kg} / \mathrm{min}$ enoximone $=1$ point \\
\hline $0.01 \mu \mathrm{g} / \mathrm{kg} /$ min noradrenaline $=1$ point \\
\hline
\end{tabular}

feasibility of treating underlying cause (e.g., revascularization if it is acute MI, antidote for poisoning, etc.) (-Fig. 1).

Despite the best conservative management, if the patient fails to stabilize in 4 to 6 hours, ECMO should be considered, if selection criteria meet and after ruling out contraindication. Most patients are put on venoarterial (VA) ECMO and a few might require hybrid procedure such as veno-arterialvenous (VAV) ECMO. Usually the femoral artery and femoral vein are cannulated for peripheral ECMO, and in case of postcardiotomy $\mathrm{CS}$, one can continue with central cannulation (i.e., RA and aorta). ECMO flow should be adjusted to achieve the target mean arterial pressure of $70 \mathrm{~mm} \mathrm{Hg}$, venous saturation of 70 , and lactates $<2 \mathrm{mmol} / \mathrm{L}$. Once good tissue perfusion is established, inotropes and vasoconstrictors are weaned off. Heparinization should be done and

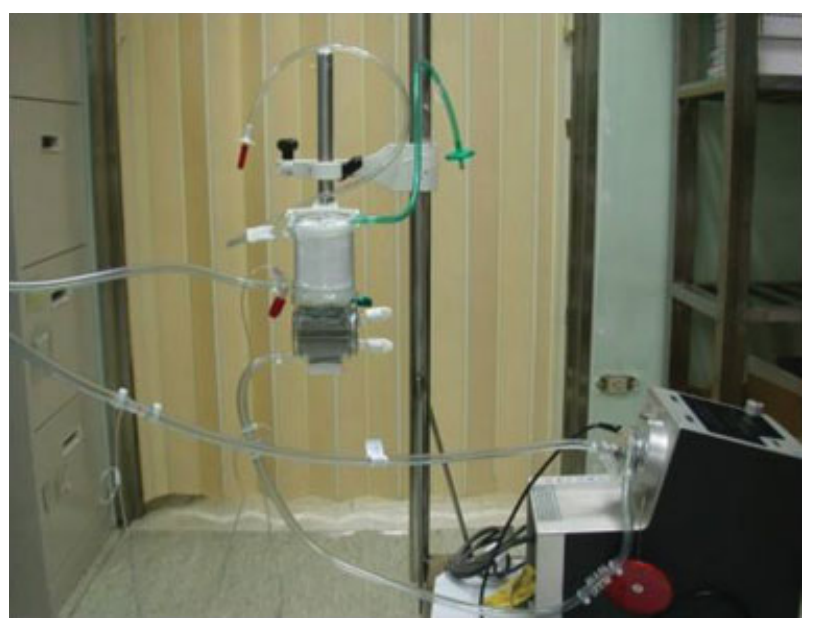

Fig. 1 ECMO circuit.

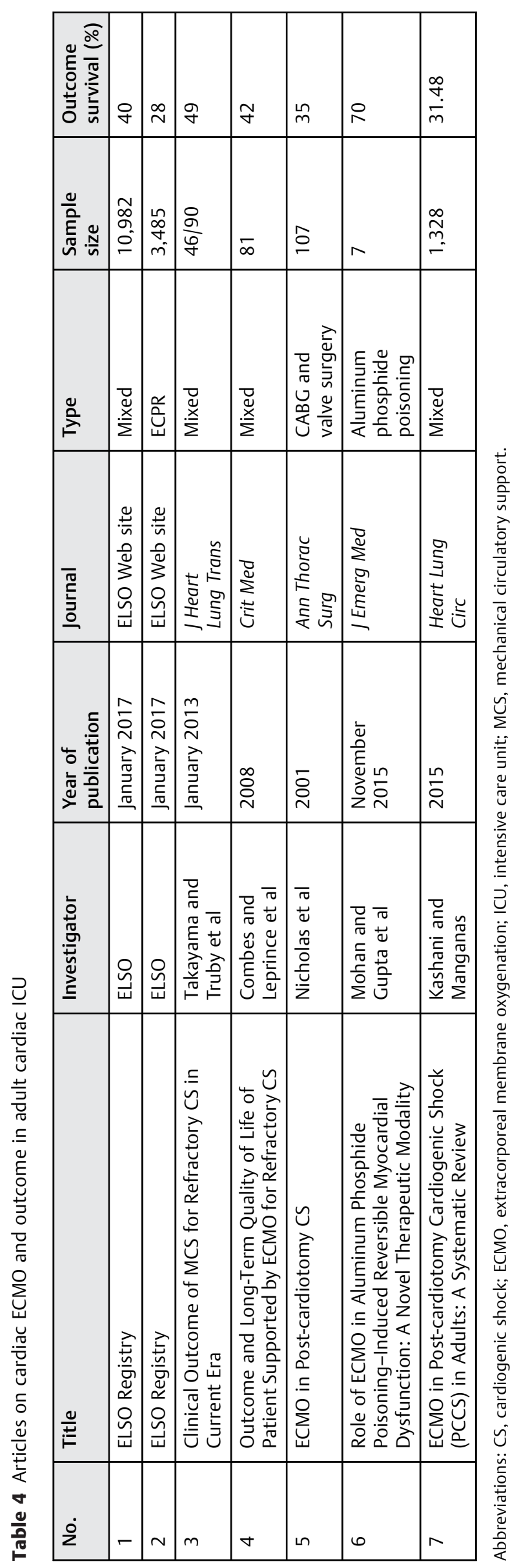




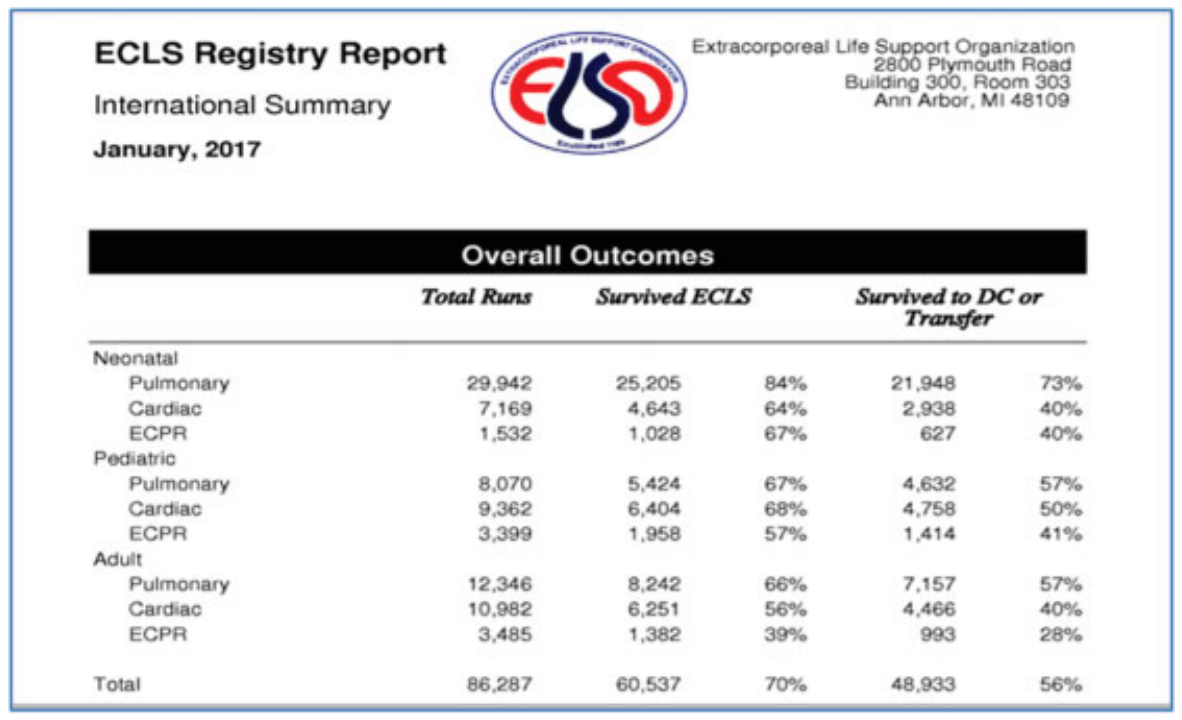

Fig. 2 Extracorporeal life support Organization (ELSO) International Registry, January 2017. DC, ECLS, extracorporeal life support; ECPR, extracorporeal cardiopulmonary resuscitation.

titrated to maintain activated clotting time (ACT) of around 160 to 180 seconds. Precaution should be taken to avoid full bypass as that will lead to cardiac stunning and even lungs need some blood flow to survive. Ventilator should be reduced to rest ventilator settings. The rest ventilator settings for cardiac ECMO include pressure control mode, peak pressure $<26$, relative risk (RR) $12, \mathrm{FiO}_{2}$ of 40 to $50 \%$, and positive end-expiratory pressure (PEEP) $10 \mathrm{~cm} \mathrm{H}_{2} \mathrm{O}$.

If the cardiac stunning occurs, LA or LV vent has to be considered or atrial septoplasty. Another alternative is to vent through pigtail catheter. ${ }^{10}$ Cardiac stunning can be diagnosed with pulse pressure $<10 \mathrm{~mm} \mathrm{Hg}$. The indication for LA or LV venting will be pulmonary edema on X-ray or Echo showing LV dilation.

Once the patient starts showing good pulsatility (pulse pressure $>40$ ), Echo showing good ejection fraction (EF) $>30$, and mean arterial pressure of $>70 \mathrm{~mm} \mathrm{Hg}$ with minimal inotropic support and minimal ECMO flow, the authors can consider a trial off and decannulation. ${ }^{11}$

\section{Outcome of ECMO in Cardiogenic Shock}

There are various papers on outcome of ECMO in CS with varied etiology (-Table 4). ECMO support can rescue $40 \%$ of patients with otherwise fatal CS (mortality without ECMO is $>80 \%$ ). As per ELSO data January 2017, 10,982 patients were reported in adult cardiac ECMO, out of whom 56\% survived ECLS and $40 \%$ survived to discharge ( - Fig. 2). Of 3,485 patients reported in extracorporeal cardiopulmonary resuscitation (ECPR), $28 \%$ survived to discharge. ECMO initiated late with cardiopulmonary resuscitation (CPR) during initiation or after renal or hepatic failure carried higher risks of ICU death, whereas fulminant myocarditis had a better prognosis. ${ }^{12}$ Independent predictors of ICU death evaluated at the time of ECMO onset included female sex, CPR during device insertion, prothrombin activity $50 \%$, and 24 -hour urine output $500 \mathrm{~mL}^{12}$
Even the authors experience that 33 patients on cardiac ECMO have had survival of $40 \%$ in the past 5 years. In this study, the authors found that lactate trend and inotropic score trend remained as good prognostic marker. Decrease in inotropic score and lactate level by $75 \%$ in first 24 hours of initiation of ECMO has good survival chance. The newer scoring system named SAVE score (its online calculator [www.save-score.com]) offers a validated tool to predict survival for patients receiving ECMO for refractory CS. ${ }^{13}$ A SAVE score of 0 was approximately equivalent to $50 \%$ survival with positive scores representing higher chances of survival and negative scores representing lower chances of survival ${ }^{13}$ ( - Table 4 ).

\section{Conclusion}

ECMO remains as a mainstay of therapy in CS of varied etiology. It is safe and has reasonably good outcome if initiated on time. The usual duration of ECMO in cardiac ICU is 5 to 7 days ( \pm 3 days). It is still being underutilized, probably due to lack of awareness, lack of experience, and the associated cost.

\section{References}

1 Reynolds, et al. Incidence of cardiogenic shock. Circulation 2008; 117:686-697

2 Thiele H, Zeymer U, Neumann FJ, et al; IABP-SHOCK II Trial Investigators. Intraaortic balloon support for myocardial infarction with cardiogenic shock. N Engl J Med 2012;367(14):1287-1296

3 Chen JS, Ko WJ, Yu HY, et al. Analysis of the outcome for patients experiencing myocardial infarction and cardiopulmonary resuscitation refractory to conventional therapies necessitating extracorporeal life support rescue. Crit Care Med 2006;34(04):950-957

4 Schwarz B, Mair P, Margreiter J, et al. Experience with percutaneous venoarterial cardiopulmonary bypass for emergency circulatory support. Crit Care Med 2003;31(03):758-764 
5 Asaumi Y, Yasuda S, Morii I, et al. Favourable clinical outcome in patients with cardiogenic shock due to fulminant myocarditis supported by percutaneous extracorporeal membrane oxygenation. Eur Heart J 2005;26(20):2185-2192

6 Doll N, Kiaii BB, Fabricius AM, et al. Intraoperative left atrial ablation (for atrial fibrillation) using a new argon cryocatheter: early clinical experience. Ann Thorac Surg 2003;76(05):1711-1715, discussion 1715

7 Massetti M, Tasle M, Le Page O, et al. Back from irreversibility: extracorporeal life support for prolonged cardiac arrest. Ann Thorac Surg 2005;79(01):178-183, discussion 183-184

8 Chen YS, Chao A, Yu HY, et al. Analysis and results of prolonged resuscitation in cardiac arrest patients rescued by extracorporeal membrane oxygenation. J Am Coll Cardiol 2003;41(02):197-203
9 Goyal V, Oza P. Indication \& Selection Criteria. ECMO-Practical Manual. 2010;1(01):1-8

10 Barbone A, Malvindi PG, Ferrara P, Tarelli G. Left ventricle unloading by percutaneous pigtail during extracorporeal membrane oxygenation. Interact Cardiovasc Thorac Surg 2011;13(03):293-295

11 Goyal V, Oza P. Veno Arterial ECMO. ECMO-Theoretical Manual. 2012;2(06):69-76

12 Combes A, Leprince P, Luyt CE, et al. Outcomes and long-term quality-of-life of patients supported by extracorporeal membrane oxygenation for refractory cardiogenic shock. Crit Care Med 2008; 36(05):1404-1411

13 Schmidt M, Burrell A, Roberts L, et al. Predicting survival after ECMO for refractory cardiogenic shock: the survival after venoarterial-ECMO (SAVE)-score. Eur Heart J 2015;36(33):2246-2256 